\title{
Orbit orientation and eye morphometrics in giraffes (Giraffa camelopardalis)
}

\author{
G. Mitchell ${ }^{1,2 *}$, D.G. Roberts', S.J. van Sittert' \& J.D. Skinner ${ }^{1, \dagger}$ \\ ${ }^{1}$ Centre for Veterinary Wildlife Studies, University of Pretoria, South Africa, and \\ ${ }^{2}$ Department of Zoology and Physiology, University of Wyoming, U.S.A.
}

Received 13 May 2013. Accepted 10 September 2013

\begin{abstract}
Giraffe are thought to have excellent vision. We measured eye size, orbit orientation and retina surface area in 27 giraffes of both sexes ranging in age from neonates to mature adults ( $>10 \mathrm{yrs}$ ), to assess how it changes with growth, whether their eye anatomy correlates with their apparently excellent vision and lifestyle, and we have compared our findings with those for other large mammals to assess whether giraffe eye anatomy is unique. We found that giraffe eye volume increases from $33 \mathrm{~cm}^{3}$ at birth to approximately $65 \mathrm{~cm}^{3}$ in adults. The focal (axial) length increases from c. 40 to $48 \mathrm{~mm}$ in adults and retina surface area from c. $3000 \mathrm{~mm}^{2}$ at birth to $4320 \mathrm{~mm}^{2}$ in adults. The orbital axis angle at birth is $c .73^{\circ}$ and the horizontal visual field mainly monocular and panoramic. With age the axis angle becomes more acute to $c .50^{\circ}$ in adults and the visual field more binocular, changes that occur concurrently with increasing neck length. These results show that the giraffe eye and retinal surface area are larger than in all other ungulates, and their visual fields more binocular, attributes which are consistent with the idea that they have excellent vision.
\end{abstract}

Key words: giraffe, eye size, orbit orientation, visual acuity.

\section{INTRODUCTION}

The preferred habitat of giraffes is woodland savanna. In this habitat, group sizes are small, the home range is larger than predicted from metabolic needs (Jetz et al. 2004), and distances between group members are large: most groups (75\%) comprise fewer than six individuals and intraspecific distances within the group can be $1 \mathrm{~km}$ (Dagg \& Foster 1976). Small group size means that the 'many eyes' vigilance a large herd provides is not available to giraffe and large intraspecific distances within a group probably require high visual acuity (Kiltie 2000). Thus giraffes should be able to detect movement, shapes and coat patch patterns at relatively long distances if they are to achieve predator avoidance, detection of competitors, mate selection and group member contact in their habitat. Of course, hearing and olfaction may also be important, although as giraffe do not have the larynx anatomy necessary to produce sounds (Harrison 1980) intraspecific communication by sounds is unlikely. Their olfactory acuity is unknown, but as the skin of adult males is known to emit a strong scent (Wood \& Weldon 2002), odour may well be an important communication signal.

It is a commonplace opinion that giraffes (Giraffa

${ }^{\dagger}$ Deceased 28 August 2011

*Author for correspondence. E-mail: mitchg@uwyo.edu camelopardalis) have excellent vision. The ancient Egyptians used sr meaning 'to foretell' as the name for giraffe alluding to their apparent ability to detect, well in advance of other species, changes occurring in their environment (Gardiner 1927), and giraffe visual excellence is legendary amongst game wardens (e.g. Bryden 1899). Giraffes can detect movement at a distance of at least $2 \mathrm{~km}$ (Dagg \& Foster 1976) and distinguish colours (Backhaus 1959). A morphological basis for these attributes partly resides in the composition of their retina of which we could find one study (Schiviz et al. 2008). As in all artiodactyls the retina of giraffes contains two types of cone which provide the capacity for dichromatic colour vision. It also has the most highly developed retinal topography of all artiodactyls examined thus far. A visual streak - an elongated area of high density of cones - typically lies horizontally at the level of the optic disc. In giraffes higher cone densities extend at both ends of the streak dorsally to form a complete arch reaching from the temporal to the nasal retinal visual fields. The dorsal extension appears to be an adaptation to height, and its presence means that when giraffes look down, images reaching the eye at a steep angle project onto areas of high cone density.

The gross anatomy of the giraffe eye is less well studied. The shape of the lens is not known. Johnson 
(1901) reported that the divergence of the optical axes was $72^{\circ}$. Ritland (1982) reported an axial length of $38.8 \mathrm{~mm}$ while Howland et al. (2004) reported an axial length of $48.147 \mathrm{~mm}$ in a $1486.5137 \mathrm{~kg}$ [sic] giraffe. Burton (2006) reported an eye mass of $63.5 \mathrm{~g}$ in a $1220 \mathrm{~kg}$ giraffe. We report here a more complete analysis of the morphometrics of the giraffe eye and orbit to establish whether the gross anatomy is consistent with their supposed excellent vision. Our hypothesis was that if giraffe vision is as good as reports suggest then eye and retina size and their fields of vision should support the folklore and the visual requirements imposed by their lifestyle.

To test this hypothesis we have measured eye mass and volume as eye size determines retina size and focal length, and the size of the image projected onto the retina, on all of which visual acuity depends. Retina size determines the number of photoreceptors across which the image is spread and thus confers the ability to see as distinct objects that are closely spaced (Ross \& Kirk 2007). We also measured the angle that the orbit subtends with the skull midline as a method for assessing the extent to which the horizontal visual fields of giraffe are panoramic, monocular or binocular. Finally, we have used published data for other mammals to make additional comparisons. Our data came from giraffes ranging in size from young to mature adults so we have been able to correlate changes in eye and orbit anatomy with growth.

\section{MATERIALS \& METHODS}

Data were obtained from 27 giraffes that had been culled in southeastern Zimbabwe. The eyes and brain were removed from each animal and brain mass was measured directly after its removal from the cranium after which the skulls were boiled and dissected clean of non-bony tissue.

\section{Eye measurements}

Right and left eye masses were measured after the eyes had been dissected free of adnexal tissue. Eye volume was measured by displacement of water in a volumetric flask and also was calculated from its external diameters. The eye was assumed to have three diameters, and each eye had its maximum external dorsoventral, lateral and anteroposterior diameters measured. The anteroposterior diameter of the eye was assumed to be the same as its axial length although correctly the axial length is the distance from the posterior

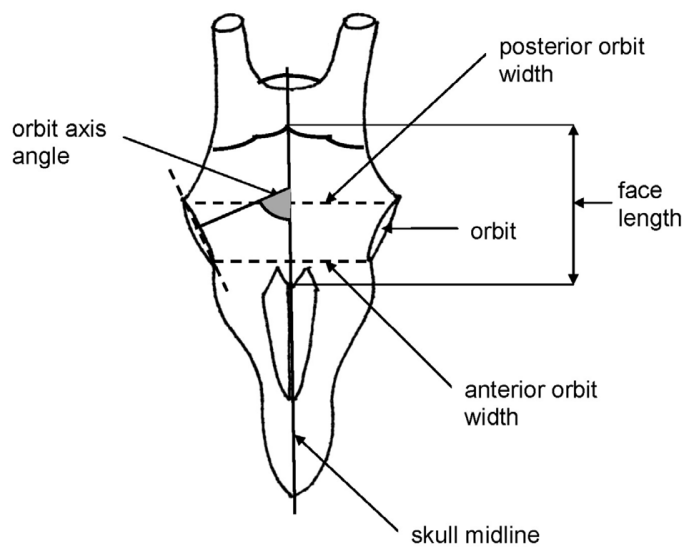

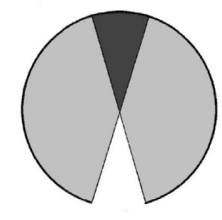

A

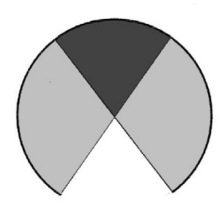

B
Fig. 1. Dorsal aspect of the skull of a giraffe showing the measurements made to determine face length and width and orbit orientation (see text) and diagrams showing the estimated changes in monocular (pale grey) and binocular (dark grey) fields of vision throughout life: $A=$ neonate and $B=$ mature $(>1000 \mathrm{~kg})$ giraffe. Note that in adult giraffe the horizontal field of vision is less monocular and more binocular than it is in neonatal animals, and the total field of vision is reduced.

surface of the cornea to the retina and thus will be somewhat shorter than the external anteroposterior diameter. We calculated the surface area of the retina using Robb's equation (Robb 1982):

$$
A=\pi H D,
$$

where $A$ is surface area $\left(\mathrm{mm}^{2}\right), H(\mathrm{~mm})$ the distance from the ora serrata to the posterior pole of the retina and $D(\mathrm{~mm})$ the equatorial diameter. We estimated $H$ as the (anteroposterior diameter of the eye $\times 0.57$ ) following Schiviz et al. (2008), and $D$ from the mean of the dorsoventral (vertical) and lateral (horizontal) diameters of the eye. The mean of the measurements made on the left and right eyes was used in analyses.

\section{Orbit measurements}

To estimate the angle that the orbit forms with the skull midline (the orbital axis) the dimensions measured (see Fig. 1) were (a) face length, defined as the distance from the articulation of the frontal with the occipital bones to their articulation with the nasal bones, (b) face width, defined as the 
Table 1. The allometry of the eye, skull and brain of 27 giraffes of both sexes, ranging from neonates to mature animals. $\mathrm{M}_{\mathrm{b}}=$ body mass; Brain = brain mass.

\begin{tabular}{llrr}
\hline Variable & Allometric equation & $R^{2}$ & $\begin{array}{r}\text { 95\% Confidence } \\
\text { limits of the slope }\end{array}$ \\
\hline Anterior orbit width $(\mathrm{mm})$ & & 0.7933 & 0.18 to 0.28 \\
Posterior orbit width $(\mathrm{mm})$ & $39.7 \mathrm{M}_{\mathrm{b}}{ }^{0.23}$ & 0.9144 & 0.23 to 0.30 \\
Orbit dorso-ventral diameter $(\mathrm{mm})$ & $45.7 \mathrm{M}_{\mathrm{b}}{ }^{0.26}$ & 0.9368 & 0.13 to 0.16 \\
Orbit lateral diameter $(\mathrm{mm})$ & $24.6 \mathrm{M}_{\mathrm{b}}{ }^{0.14}$ & 0.7626 & 0.09 to 0.15 \\
Orbit axis angle $(\mathrm{deg})$ & $31.6 \mathrm{M}_{\mathrm{b}}{ }^{0.12}$ & 0.3746 & -0.05 to -0.14 \\
Binocular overlap $(\mathrm{deg})$ & $107 \mathrm{M}_{\mathrm{b}}{ }^{-0.09}$ & 0.4256 & 0.10 to 0.29 \\
Eye mass $(\mathrm{g})$ & $16.4 \mathrm{M}_{\mathrm{b}}{ }^{0.20}$ & 0.9479 & 0.25 to 0.30 \\
Eye volume $(\mathrm{ml})$ & $9.1 \mathrm{M}_{\mathrm{b}}{ }^{0.28}$ & 0.9235 & 0.25 to 0.31 \\
Eye dorso-ventral diameter $(\mathrm{mm})$ & $8.8 \mathrm{M}_{\mathrm{b}}{ }^{0.28}$ & 0.9278 & 0.08 to 0.10 \\
Eye lateral diameter $(\mathrm{mm})$ & $26.3 \mathrm{M}_{\mathrm{b}}{ }^{0.09}$ & 0.9007 & 0.08 to 0.10 \\
Eye antero-posterior diameter $(\mathrm{mm})$ & $26.7 \mathrm{M}_{\mathrm{b}}{ }^{0.09}$ & 0.8821 & 0.08 to 0.11 \\
Retina surface area $\left(\mathrm{mm}{ }^{2}\right)$ & $24.3 \mathrm{M}_{\mathrm{b}}{ }^{0.09}$ & 0.8821 & 0.16 to 0.22 \\
Brain mass $(\mathrm{g})$ & $1153 \mathrm{M}_{\mathrm{b}}{ }^{0.19}$ & 0.7182 & 0.11 to 0.19 \\
Eye mass $(\mathrm{g})$ & $237.0 \mathrm{M}_{\mathrm{b}}^{0.15}$ & 0.6822 & 0.94 to 1.67 \\
Retina surface area $\left(\mathrm{mm}^{2}\right)$ & $01 \mathrm{Brain}^{1.31}$ & 0.6620 & 0.61 to 1.11 \\
\hline
\end{tabular}

distance between the postero-lateral margins of the orbits (posterior orbital width (POW)), (c) orbit width which was calculated from the difference between POW and the distance between the anterolateral margins of the orbits (anterior orbital width (AOW)), and (d) the face length: width ratio. In addition we measured the height or dorsoventral diameter of the orbit between the dorsal margin formed by the frontal bone and the ventral margin on the zygomatic arch, and its horizontal or lateral diameter extending from the medial margin formed by the frontal and lachrymal bones to the lateral margin on the posterior rim of the zygomatic arch. The angle of the orbital axis was the angle at which a line running perpendicular to the anterior and posterior orbit margins transected the skull midline (Fig. 1). This angle is the cosine of (orbit width $\div$ orbit lateral diameter). From the orbital axis angle the area of the field of vision that is covered by both eyes (i.e. is binocular) can be estimated from the equation:

$$
2^{*}(90 \text {-orbital axis angle). }
$$

The mean of the measurements made on the left and right orbits was used in analyses.

\section{Growth analysis}

The relationships between body dimensions and organ dimensions during growth can be described through power functions of the form

$$
y=a M_{b}^{b} \text {, }
$$

where $a$ is the intercept, $M_{\mathrm{b}}$ body mass in $\mathrm{kg}$ and $b$ the slope. The data were log transformed and used to develop equations of intercepts and exponents for the slopes; with eye morphology and body mass the covariates. Confidence limits $(95 \%)$ were determined using the two variable geometric regression calculator available at www.benchmark calculations.com, which also generates trend curves for the relationship between covariates and calculates the correlation coefficient. Average values for variables were calculated from all values obtained and differences between them, and for them between the sexes, were evaluated by the two-tailed $t$-test assuming unequal variances. $P$ values $<0.05$ were regarded as significant.

\section{RESULTS}

Data were obtained from 27 giraffes of both sexes (10 females, 17 males) and covering a full spectrum of body masses that ranged from 179 to $1440 \mathrm{~kg}$. Body mass increases with age. At birth a giraffe weighs $100 \mathrm{~kg}$. At two years of age body mass is c. $450 \mathrm{~kg}$, at four years of age c. $700 \mathrm{~kg}$, at six years of age c. $1000 \mathrm{~kg}$ (= maximum female body mass) with males reaching their maximum body mass of about $1500 \mathrm{~kg}$ at $c .10$ years of age. In our sample eight giraffes had a body mass of 100 to $450 \mathrm{~kg}$ (179-377 kg), five a body mass of 450 to $700 \mathrm{~kg}$ $(543-681 \mathrm{~kg})$, nine a body mass of 700 to $1000 \mathrm{~kg}$ $(727-978 \mathrm{~kg})$ and five of the males had a body mass of more than $1000 \mathrm{~kg}$ (1023-1440 kg). No sexual dimorphism was expected and no significant differences could be found between the sexes for any of the variables analysed, and data were pooled. The allometry of the eye of giraffes is summarized in Table 1. All the correlation coefficients between body mass and the variables 
Table 2. The mean \pm S.D. and range for eye, skull and brain variables in 27 giraffes.

\begin{tabular}{|c|c|}
\hline Variable & Giraffe \\
\hline Anterior orbit width $(\mathrm{mm})$ & $\begin{array}{l}178.7 \pm 26.9 \\
\text { (Range }=124-218)\end{array}$ \\
\hline Posterior orbit width (mm) & $\begin{array}{l}245.7 \pm 37.0 \\
\text { (Range }=177-292)\end{array}$ \\
\hline Face length: width ratio & $\begin{array}{l}0.71 \pm 0.05 \\
(\text { Range }=0.63-0.84)\end{array}$ \\
\hline Orbit dorsoventral diameter (mm) & $\begin{array}{l}60.6 \pm 5.2 \\
\text { (Range }=51.4-71.5)\end{array}$ \\
\hline Orbit lateral diameter (mm) & $\begin{array}{l}68.6 \pm 5.2 \\
(\text { Range }=55.3-79.5)\end{array}$ \\
\hline Orbit axis angle (deg) & $\begin{array}{l}60.3 \pm 5.2 \\
\text { (Range = 73.3-46.1) }\end{array}$ \\
\hline Binocular overlap (deg) & $\begin{array}{l}59.5 \pm 10.4 \\
(\text { Range }=33.3-87.9)\end{array}$ \\
\hline Eye mass $(\mathrm{g})$ & $\begin{array}{l}53.4 \pm 8.6 \\
\text { (Range }=38.0-67.0)\end{array}$ \\
\hline Eye volume (ml) & $\begin{array}{l}53.0 \pm 8.7 \\
(\text { Range }=37.6-64.0)\end{array}$ \\
\hline Eye dorsoventral diameter (mm) & $\begin{array}{l}46.8 \pm 2.6 \\
\text { (Range }=42.0-50.4 \text { ) }\end{array}$ \\
\hline Eye lateral diameter (mm) & $\begin{array}{l}47.3 \pm 2.6 \\
\text { (Range }=41.8-50.8)\end{array}$ \\
\hline Eye antero-posterior diameter (mm) & $\begin{array}{l}44.1 \pm 2.6 \\
\text { (Range }=38.5-47.7)\end{array}$ \\
\hline Retina surface area $\left(\mathrm{mm}^{2}\right)$ & $\begin{array}{l}3724 \pm 405 \\
\text { (Range }=2967-4320)\end{array}$ \\
\hline Brain mass $(\mathrm{g})$ & $\begin{array}{l}629.1 \pm 66.5 \\
\text { (Range }=494-749)\end{array}$ \\
\hline Eye mass: brain mass (\%) & $\begin{array}{l}8.5 \pm 0.9 \\
\text { (Range = 6.9-11.1) }\end{array}$ \\
\hline
\end{tabular}

measured were significant. Table 2 shows average values and the range for the variables measured and Table 3 shows a comparison between giraffe eyes and those of some large land mammals. Figure 2 shows the trend curves for the four principal variables analysed in this study.

Mean eye mass $(53.4 \pm 8.6 \mathrm{~g})$, displacement volume $\left(53.0 \pm 8.7 \mathrm{~cm}^{3}\right)$ and the geometric (spherical) volume of the eye $\left(51.6 \pm 7.9 \mathrm{~cm}^{3}\right)$ calculated from the mean of its three diameters were not signifi- cantly different. This result confirmed that the eyeball can be considered to be roughly spherical although the anteroposterior diameter (axial length; $44.1 \pm 2.6 \mathrm{~mm}$; range $38.5-47.7 \mathrm{~mm}$ ) was significantly shorter than either the lateral $(47.3 \pm$ $2.6 \mathrm{~mm}$; range $41.8-50.8 \mathrm{~mm}$ ) or dorsoventral diameter $(46.8 \pm 2.6 \mathrm{~mm}$; range $42.0-50.4 \mathrm{~mm})$, the lengths of which did not differ significantly. Eye density was $1.009 \pm 0.032 \mathrm{~g} / \mathrm{ml}$ and the ratio of eye mass to brain mass was $8.5 \pm 0.9 \%$. During growth, eye mass, volume and axial length increased significantly $(P<0.05$; Fig. $2 \mathrm{~A}, \mathrm{~B})$. We found average retinal surface area to be $3724 \pm 405 \mathrm{~mm}^{2}$ (mean \pm S.D.; range $2967-4320 \mathrm{~mm}^{2}$ ) and the rate of increase of retina surface area followed that of eye size.

The shape of the rim of the orbit was not circular: the dorsoventral diameter was significantly smaller than the lateral diameter with the lateral diameter being approximately 1.13 -fold longer. Giraffe develop broad short faces during growth (the length to width ratio becomes less than 1). The broadening of the face had the effect that the orbit axis angle during growth became more acute and decreased from $c .75^{\circ}$ in neonates to $c .50^{\circ}$ in mature giraffe (Fig. 2C). The area of the binocular field of vision increased during growth from $33^{\circ}$ in neonates to $70^{\circ}$ in mature adults (Figs $1 \& 2 \mathrm{C}$ ).

\section{DISCUSSION}

In this study we tested the idea that the macroanatomy of the giraffe eye has evolved in ways that contribute to excellent vision. We have used eye size, retina surface area, and the anteroposterior diameter of the eye (as a proxy for axial or focal length) as measures of visual acuity (Howland et al. 2004) and we compared giraffe eye morphology with that of other large land mammals to assess if giraffe eye anatomy differs significantly from them. Our data show that the eyes of giraffes are larger, have a larger retina surface area, and a longer focal length, than all land mammals including elephants (Loxodonta africana). Elephants have

Table 3. Comparison of eye mass, axial length, and the estimated number of cones in the retina in some large land mammals with those of giraffe (values in brackets) of the same body mass $\left(M_{b}\right)$; data on giraffe are from this study. Data on eye mass are from Burton (2006) and on axial length from Howland et al. (2004).

\begin{tabular}{lrcrcc}
\hline Species & $\mathrm{M}_{\mathrm{b}}(\mathrm{kg})$ & Eye mass $(\mathrm{g})$ & $\mathrm{M}_{\mathrm{b}}(\mathrm{kg})$ & $\begin{array}{c}\text { Axial length } \\
(\mathrm{mm})\end{array}$ & $\begin{array}{c}\text { Estimated number } \\
\text { of cones }\left(\times 10^{6}\right)\end{array}$ \\
\hline Rhino, Rhinoceros bicornis & 764 & $11.3(58.4)$ & 764 & $23.6(44.2)$ & $32(115)$ \\
Hippo, Hippopotamus amphibus & 947 & $19.4(62.0)$ & 1351 & $29(46.5)$ & $49(120)$ \\
Buffalo, Syncerus caffer & 666 & $31.2(56.2)$ & 759 & $32(44.1)$ & $59(112)$ \\
Elephant, Loxodonta africana & 6654 & $58(-)$ & 4755 & $51(-)$ & $75(-)$ \\
\hline
\end{tabular}



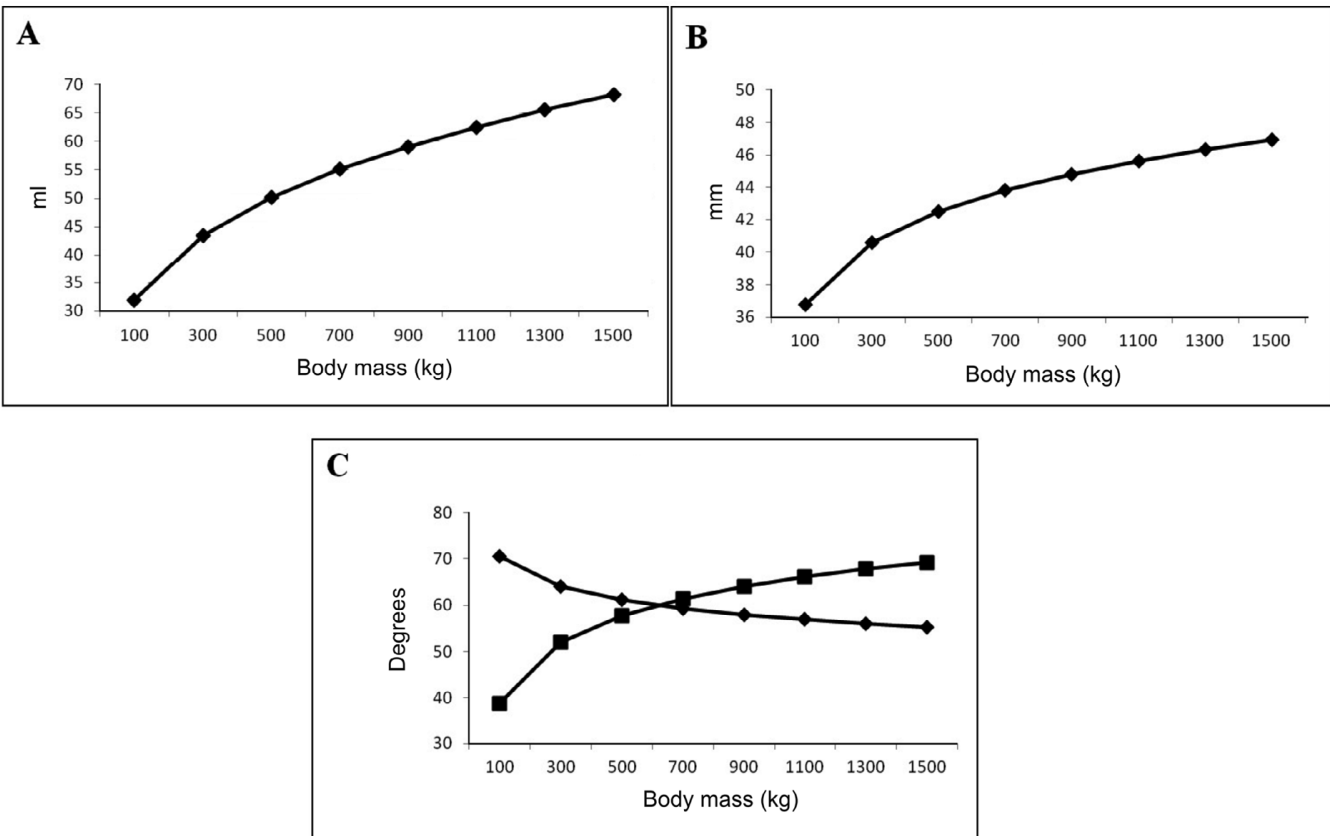

Fig. 2. Changes in eye volume (A), axial length (B) and (C) orbit axis angle $(\bullet)$ and binocular overlap ( $\mathbf{\square})$ during growth as measured by increases in body mass.

an elongated eye so their eye volume is smaller than it is in giraffes but the axial length longer. In giraffes the combination of focal length retinal surface area and cone number place them in a group of artiodactyls (with camels, Camelus sp.; eland, Taurotragus oryx; and kudu, Tragelaphus strepsiceros) that have highest resolution capacity. All these species are browsers.

We also found, like Kiltie (2000) that increases in eye size in giraffes are hypoallometric but correlate well with increases in body mass. The maximum body mass of adult giraffes (c. $1500 \mathrm{~kg}$ in males) is the highest of any terrestrial mammal after adult white rhinoceros (Ceratotherium simum; c. $2500 \mathrm{~kg}$ ) and elephants (c. $6000 \mathrm{~kg}$ ). Giraffe eye size should be large, therefore, but the question is whether their eyes are larger or smaller than expected for their body mass. We could find just one previous report of giraffe eye size. Burton (2006) reported an eye mass of $63.5 \mathrm{~g}$ in a mature $(1220 \mathrm{~kg})$ giraffe, which is similar to the eye mass we found in mature giraffes. However, using that data and eye mass and body mass $\left(\mathrm{M}_{\mathrm{b}}\right)$ data in 19 other ungulates excluding giraffe, the average eye mass of ungulates is predicted to be $3.96 \mathrm{M}_{\mathrm{b}}{ }^{0.33}$ $\left(R^{2}=0.3979\right)$ (Burton 2006). Using this relationship the eye mass of the largest giraffe in our study (body mass $=1440 \mathrm{~kg}$ ) was predicted to be $44 \mathrm{~g}$ compared to the $67 \mathrm{~g}$ we found. This analysis suggests that giraffe eyes are bigger than predicted from the relationship between eye mass and body mass in other ungulates. However, the eye is an extension of the brain and the eye mass of ungulates should be on average $9.4 \pm 4.3 \%$ of brain mass (Burton 2006). Our data show that the relationship between brain mass and eye mass is almost linear (Table 1) and that in our giraffes the eye: brain ratio was $8.5 \pm 0.9 \%$. We conclude that compared to other ungulates, giraffe eye mass is larger than expected relative to body mass but appropriate for their brain mass, with the size of the eye in an adult giraffe being the largest yet recorded for a land mammal (see Table 3).

Additionally, the eye axial length in giraffes was proportionate to body mass and similar to values previously reported. Howland et al. (2004) reported an axial length of $48 \mathrm{~mm}$ in a giraffe of body mass $1468 \mathrm{~kg}$ and Ritland (1982) an axial length of $38.8 \mathrm{~mm}$ in a giraffe, which, from our data, would have related to a body mass of about $1000 \mathrm{~kg}$. The heaviest giraffe in our series weighed $1440 \mathrm{~kg}$ and it had an eye anteroposterior diameter of $45.7 \mathrm{~mm}$. Using the data given by Howland et al. (2004) for 15 ungulates the predicted axial length $\left(A L=16.5 \mathrm{Mb}^{124} ; R^{2}=0.7299\right)$ for the giraffes in our study was $36.5 \pm 2.7 \mathrm{~mm}$ compared to the $44.1 \pm$ 
$2.6 \mathrm{~mm}$ we found. The smaller length predicted from Howland et al.'s data (2004) and reported by Ritland (1982) most likely arises from the difference in the way the lengths were measured. True axial length is the distance measured from the posterior surface of the cornea to the retina whereas our measurement was made from the anterior surface of the cornea to the posterior surface of the eyeball. The thickness of the cornea and the posterior wall of the eyeball accounts for the difference between our and Howland et al.'s (2004) values. Nevertheless our data suggest that axial length in mature giraffes is longer than any other land mammal except elephant (Table 3).

Visual acuity is also dependent on the number of cone photoreceptors present which in turn depends on retina surface area and the number of cones per $\mathrm{mm}^{2}$ of retina surface area. The average number of cones per $\mathrm{mm}^{2}$ of retina is quite constant in mammals $\left(28.2 \times 10^{3} \pm 1.3 \times 10^{3} / \mathrm{mm}^{2}\right.$; mean \pm S.E.M.; Schiviz et al. 2008). For the giraffes in this study, we estimated that on average the number of cones present in the retina was approximately 105 million (range 84-122 million) which is the highest for the artiodactyls listed by Schiviz et al. (2008) and more than in other large land mammals (Table 3). The large size, long axial length and retina anatomy of giraffe eyes are sufficient to explain the good vision that direct observation and previous reports suggest that giraffes possess (Backhaus 1959; Dagg \& Foster 1976).

Our data also indicate that the binocular field of vision of giraffes increases with growth. A likely cause of this increase is that during growth, inflation of the frontal sinuses causes the face to become increasingly broader (Singer \& Bonè 1960; Spinage 1968; Badlangana et al. 2011), accompanied by enlargement of the lachrymal bone (Owen 1838). These changes appear to push the orbit outwards and forwards, so moving the visual field from being mainly monocular to more binocular (Fig. 1B). Assuming that the orbital axis and the optical axis (the hypothetical straight line passing through the centers of curvature of the front and back surfaces of a simple lens) coincide, then the optical axis of $72^{\circ}$ reported by Johnson (1901) was measured in the eyes of a young $(<1$ year-old $)$ giraffe weighing c. $240 \mathrm{~kg}$. At birth $\left(\mathrm{M}_{\mathrm{b}}=100 \mathrm{~kg}\right)$ the orbital and optical axes are $c .73^{\circ}$, i.e. the eyes face almost laterally, a conclusion supported by our own observation of foetal and neonatal giraffes that have the long narrow faces appropriate for birth. The most acute orbit/optical axis angle we measured was $46^{\circ}$ in a mature female $\left(\mathrm{M}_{\mathrm{b}}=967 \mathrm{~kg}\right)$ which corresponds to an area of binocular field of vision of $88^{\circ}$.

The general advantage that binocular overlap confers is stereoscopic depth perception (Heesy 2004, 2008), but the advantage specifically for giraffes is not obvious. Judgment of depth and distance is a complex process involving several factors such as perspective, rate of movement of an object across a visual field, eye re-orientation, and contrast discrimination, and it is enhanced by binocular vision (Heesy 2009). In birds and amphibians the effects of binocular vision are limited to a short range ( $<1 \mathrm{~m}$, Heesy 2008). The range over which it functions in mammals is unknown, but is assumed to be limited also. The range is partly dependent on interocular distance (Heesy 2009). The interocular distance in giraffes is comparatively very large (up to $300 \mathrm{~mm}$ in a mature animal) and thus the range over which depth and distance perception is enhanced may be longer in them and may contribute to their ability to detect movement at great distances. Nevertheless, if stereopsis operates only over short distances in giraffes it, together with their ability to discriminate between closely spaced objects that the number of cones confers (Schiviz et al. 2008), may facilitate the acquisition of browse. Acquisition of browse, like judgment of depth, is a complex process relying on taste, touch and smell as well as vision. Giraffes are known to be careful browsers and good near distance depth judgment together with olfaction and touch may facilitate discrete selection of the browse that they require.

The physical attribute that is most characteristic of giraffes is their long neck. As increases in both eye size and neck length correlate with increases in body mass (Mitchell et al. 2009; this study), our data suggest that neck elongation is accompanied by changes in visual anatomy that are associated with good vision. In other words it seems as if giraffes have co-evolved good vision and a periscope-like ability to see above tree level as advantageous adaptations for the open woodland savanna environment they inhabit: these two characteristics provide an early warning system that enhances predator avoidance, and provide a means of communication between widely separated conspecifics. This conclusion is supported by scaling analyses that suggest that visual distances for intraspecific social interactions within a group should increase as visual acuity increases (Kiltie 2000). We can speculate that giraffe visual anatomy 
might have been shaped by the conditions that existed at the time when giraffes were evolving rapidly. Coincident with the radiation of Giraffa, was climate change that caused tropical vegetation to give way to the arid, open woodland savanna (Pellew 1983; Janis 1993; Mitchell \& Skinner 2003). This period was associated with the immigration of large predators into the African savanna (Janis 1993). These are circumstances in which increases in home range size, vigilance, communication and an ability to detect and avoid predators constitute significant evolutionary pressures. The advantage of good, periscope-like vision in both sexes may, therefore, have reinforced the natural selection for long necks caused by other factors.

\section{ACKNOWLEDGEMENTS}

We thank the management and staff of the Bubye Valley Conservancy, Zimbabwe, for their logistical support and sponsorship of this study, Roberto Portela-Miguez and Andy Currant at the British Museum of Natural History, Shaw Badenhorst of the Ditsong National Museum of Natural History (formerly the Transvaal Museum). Funds were provided by the Don Craib Trust (J.D.S.), from a personal research grant (J.D.S.) and the University of Wyoming (G.M.). S.J.v.S. is in receipt of the Maberly Memorial Scholarship and a University of Pretoria study bursary. Technical field support was provided by Kenneth Manyangadze, Mark Brewer, Lauren Leathem, Kyle Pearse, Declan Gallagher, Blake Wilelmi and Megan North. We also thank the reviewers for their helpful comments.

\section{REFERENCES}

BADLANGANA, N.L., ADAMS, J.W. \& MANGER, P.R. 2011. A comparative assessment of the size of the frontal air sinuses in the giraffe (Giraffa camelopardalis). Anatomical Record 294: 931-940.

BACKHAUS, D. 1959. Experimentelle Prüfung des Farbsehvermögens einer Massai-giraffe (Giraffa camelopardalis tippelskirchi Matschie 1898). Zeitschrift für Tierpsychologie 16: 468-477.

BRYDEN, H.A. 1899. Great and Small Game of Africa. Rowland Ward, London.

BURTON, R.F. 2006. A new look at the scaling of size in mammalian eyes. Journal of Zoology, London 269: 225-232.

DAGG, A.I. \& FOSTER, J.B. 1976. The Giraffe: Its Biology, Behavior, and Ecology. Van Nostrand Reinhold, New York.

GARDINER A. 1927. Egyptian Grammar. Clarendon Press, London.

HARRISON, D.F.N. 1980. Biomechanics of the giraffe larynx and trachea. Acta Otolaryngology 89: 258-264.

HEESY, C.P. 2004. On the relationship between orbit orientation and binocular visual field overlap in mammals. Anatomical Record 281A: 1104-1110.

HEESY, C.P. 2008. Ecomorphology of orbit orientation and the adaptive significance of binocular vision in primates and other mammals. Brain Behaviour and Evolution 71: 54-67.

HEESY, C.P. 2009. Seeing in stereo: the ecology and evolution of primate binocular vision and stereopsis. Evolutionary Anthropology 18: 21-35.

HOWLAND, H.C., MEROLA, S. \& BASARAB, J.R. 2004. The allometry and scaling of the size of vertebrate eyes. Vision Research 44: 2043-2065.

JANIS, C.M. 1993. Tertiary mammal evolution in the context of changing climates, vegetation, and tectonic events. Annual Review of Ecology and Systematics 24: 467-500.

JETZ, W., CARBONE, C., FULFORD, J. \& BROWN, J.H. 2004. The scaling of animal space use. Science 306: 266-268.

JOHNSON, J.L. 1901. Contributions to the comparative anatomy of the mammalian eye, chiefly based on ophthalmoscopic examination. Philosophical Transactions of the Royal Society B 194: 1-82.

KILTIE, R.A. 2000. Scaling of visual acuity with body size in mammals and birds. Functional Ecology 14: 226-234.

MITCHELL, G. \& SKINNER, J.D. 2003. On the origin, evolution and phylogeny of giraffes Giraffa camelopardalis. Transactions of the Royal Society of South Africa 58: 51-73.

MITCHELL, G., VAN SITTERT, S. \& SKINNER, J.D. 2009. Sexual selection is not the origin of long necks in giraffes. Journal of Zoology, London 278: 281-286

OWEN, R. 1838. Notes on the anatomy of the Nubian giraffe. Transactions of the Zoological Society of London 2: 217-248.

PELLEW, R.A. 1983. The giraffe and its food resource in the Serengeti. I. Composition, biomass, and production of available browse. African Journal of Ecology 21: 241-267.

RITLAND, S. 1982. The allometry of the vertebrate eye. Ph.D. dissertation, University of Chicago, Ann Arbor.

ROBB, R.M. 1982. Increase in retinal surface area during infancy and childhood. Journal of Pediatric Ophthalmology and Strabismus 19: 16-20.

ROSS, C.F. \& KIRK, E.C. 2007. Evolution of eye size and shape in primates. Journal of Human Evolution 52: 294-313.

SCHIVIZ, A.N., RUF, T., KUEBBER-HEISS, A., SCHUBERT, C. \& AHNELT, P.K. 2008. Retinal cone topography of artiodactyl mammals: influence of body height and habitat. Journal of Comparative Neurology 507: 1336-1350.

SINGER, R. \& BONÈ, E.L. 1960. Modern giraffes and the fossil Giraffids of Africa. Annals of the South African Museum 45: 375-548.

SPINAGE, C.A. 1968. Horns and other bony structures of the skull of the giraffe, and their functional significance. East African Wildlife Journal 6: 53-61.

WOOD, W.F. \& WELDON, P.J. 2002. The scent of the reticulated giraffe (Giraffa camelopardalis reticulata). Biochemical Systematics and Ecology 30(10): 913-917. 